\title{
Long-Term Depression of Intrinsic Excitability Accompanied by Synaptic Depression in Cerebellar Purkinje Cells
}

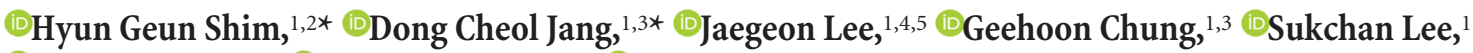

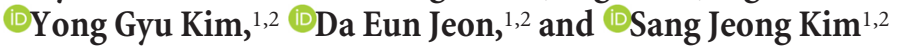 \\ ${ }^{1}$ Department of Physiology and ${ }^{2}$ Department of Biomedical Science, Seoul National University College of Medicine, 03087 Seoul, Korea, ${ }^{3}$ Department of \\ Brain and Cognitive Science, College of Science, Seoul National University, 08826 Seoul, Korea, and ${ }^{4}$ Department of Herbal Resources and ${ }^{5} \mathrm{Hanbang}$ \\ Body-fluid Research Centre, Wonkwang University, 54538 Iksan, Korea
}

Long-term depression (LTD) at the parallel fiber (PF)-to-cerebellar Purkinje cell (PC) synapse is implicated in the output of PCs, the sole output of the cerebellar cortex. In addition to synaptic plasticity, intrinsic excitability is also one of the components that determines PC output. Although long-term potentiation of intrinsic excitability (LTP-IE) has been suggested, it has yet to be investigated how PF-PC LTD modifies intrinsic excitability of PCs. Here, we show that pairing of the PF and climbing fiber (CF) for PF-PC LTD induction evokes LTD-IE in cerebellar PCs from male C57BL/6 mice. Interestingly, this intrinsic plasticity showed different kinetics from synaptic plasticity, but both forms of plasticity share $\mathrm{Ca}^{2+}$ signaling and protein kinase $\mathrm{C}$ pathway as their underlying mechanism. Although smallconductance $\mathrm{Ca}^{2+}$-activated $\mathrm{K}^{+}$channels play important roles in LTP-IE, no direct implication has been found. After PF-PC LTD induction, neither the temporal summation of dendritic EPSP nor the power of spike frequency adaptation is changed, indicating that cerebellar LTD executes the information processing in a quantitative way without quality changes of synaptic integration and generation of output signals. Our results suggest that LTD-IE may have a synergistic effect with synaptic depression on the total net output of neurons by amplifying the modification of PF synaptic transmission.

Key words: cerebellar Purkinje cells; flocculus; intrinsic excitability; long-term depression

Significance Statement

Although the output of Purkinje cells (PCs) is a critical component of cerebellum-dependent learning and memory, the changes of PC excitability when synaptic LTD occurs are unclear. Here, we show that the induction of PF-PC LTD evokes LTD-IE in PCs. Our observation complements previous intrinsic plasticity phenomenon of long-term potentiation of intrinsic excitability (LTP-IE), providing evidence for the idea that intrinsic plasticity has bidirectionality as synaptic plasticity. LTD-IE occurs together with synaptic LTD and both phenomena are dependent on the $\mathrm{Ca}^{2+}$ signaling pathway. Furthermore, our findings raise the prospect that this synaptic and intrinsic plasticity acts synergistically in PCs to modify neuronal activity in the same direction when learning occurs.

\section{Introduction}

A long-standing question in the neuroscience field is how the brain stores information from the surroundings and subse-

Received Nov. 8, 2016; revised April 11, 2017; accepted April 26, 2017.

Author contributions: H.G.S., D.C.J., and S.J.K. designed research; H.G.S., D.C.J., and J.L. performed research; H.G.S., D.C.J., Y.G.K., and D.E.J. contributed unpublished reagents/analytic tools; H.G.S., D.C.J., and J.L. analyzed data; H.G.S., D.C.J., G.C., S.L., and S.J.K. wrote the paper.

This study was supported by the National Research Foundation of Korea (NRF Grant funded by the Korean Govemment MSIP 2012R1A5A2A44671346and Global Ph.D. Fellowship Programme Grant NRF-2013H1A2A1034318). We thank Yoonseon Oh for building an AP-analyzing MATLAB code; Yong-Seok Lee, Misun Mun, Seung Ha Kim, and Jaehee Kim for proofreading the manuscript; Soo Yong Kim for technical assistance; and Kyung-Ran Kim for revising the data. quently modifies the weight of input and output signals to adjust behavior. To answer this question, activity-dependent changes in synaptic transmission such as long-term potentiation (LTP) and long-term depression (LTD) have been studied intensively as the cellular mechanisms of this phenomenon (Kandel et al., 2014). Changes in intrinsic excitability of neurons also have been impli-

The authors declare no competing financial interests.

*H.G.S. and D.C.J. contributed equally to this work.

Correspondence should be addressed to Sang Jeong, Kim Department of Physiology, Seoul National University College of Medicine, 03087 Seoul, Korea. E-mail: sangjkim@snu.ac.kr.

DOI:10.1523/JNEUROSCI.3464-16.2017

Copyright $\odot 2017$ the authors $\quad 0270-6474 / 17 / 375659-11 \$ 15.00 / 0$ 
cated in the output of neuron and finally memory processing as well (Straka et al., 2005). A recent study showed that synaptic LTP of cerebellar PC accompanies potentiation of intrinsic excitability (Belmeguenai et al., 2010). This modification of excitability indeed complements synaptic inputs and generates synergistically a total net output of the neurons.

The classical view of cerebellum-dependent motor learning has been focused on the synaptic plasticity between parallel fibers (PFs) and Purkinje cells (PCs) within the cerebellar cortex (Ito, 1982; De Zeeuw et al., 1998; Hansel et al., 2006). Emerging evidence has shown that synaptic plasticity can be induced at multiple sites (Gao et al., 2012). Recently, Ito (2013) suggested that fast adaptation of vestibulo-ocular reflex (VOR) learning is based on the LTD at PF-PC synapses and slow adaptation is derived from mossy fiber (MF)-to-vestibular nucleus (VN) neuron synapses. Indeed, PCs integrate the input from the precortical region and generate an output signal, thereby transferring the memory trace to the postcortical area (Kassardjian et al., 2005; Okamoto et al., 2011). Therefore, the activity of cerebellar PCs plays a central role in the acquisition of the VOR beyond synaptic plasticity (Kassardjian et al., 2005; Okamoto et al., 2011). Moreover, the spiking activity of PCs has a large impact on the cerebellar-motor memory consolidation (Wulff et al., 2009; Galliano et al., 2013), indicating that the output of PCs may play a critical role in memory processing within the motor circuitry. Therefore, observation of an activity-dependent modulation of intrinsic excitability will provide insight into understanding information processing and memory storage in cerebellar circuits beyond synaptic plasticity in terms of motor learning. Despite the physiological significance of intrinsic plasticity, characteristics of intrinsic plasticity accompanied by PF-PC LTD remain unclear.

Here, we address the question how intrinsic plasticity is modulated after synaptic depression of PCs by using a whole-cell patch-clamp (PC) technique from the flocculus and the vermis lobular III-V from 4- to 6-week-old male C57BL/6 mice. We found that the conjunctive activation of $\mathrm{PF}$ and climbing fiber (CF), the well known induction protocol for PF-PC synaptic LTD, induced LTD of intrinsic excitability (LTD-IE) as well. Both synaptic and intrinsic plasticity are the $\mathrm{Ca}^{2+}$ dependent and share the protein kinase C (PKC) pathway. This learned pattern of the neuronal output functions as an amplifier of depressed synaptic strength rather than modifying the strategy for input integration and output generation.

\section{Materials and Methods}

Animals and slice preparation. All animal use was in accordance with protocols approved by the Animal Care and Use Committee of Seoul National University College of Medicine. Cerebellar parasagittal slices were dissected into $250 \mu \mathrm{m}$ using a vibratome (Leica, VT1200) from anesthetized 4- to 6-week-old male C57BL/6 mice in ice-cold standard artificial CSF (aCSF) containing the following (in $\mathrm{mm}$ ): $125 \mathrm{NaCl}, 2.5$ $\mathrm{KCl}, 1 \mathrm{MgCl}_{2}, 2 \mathrm{CaCl}_{2}, 1.25 \mathrm{NaH}_{2} \mathrm{PO}_{4}, 26 \mathrm{NaHCO}_{3}$, and 10 glucose bubbled with $95 \% \mathrm{O}_{2}$ and $5 \% \mathrm{CO}_{2}$. For recovery, slices were incubated at $32^{\circ} \mathrm{C}$ for $30 \mathrm{~min}$ and then a further $1 \mathrm{~h}$ at room temperature.

Electrophysiology. Slices were put onto a submerged recording chamber on the stage of an Olympus BX50WI microscope, perfused with aCSF, and kept in place with a nylon-strung platinum anchor. All recordings were performed using EPC9 (HEKA Elektronik) and multiclamp 700B PC amplifier (Molecular Devices) with a sampling frequency of 20 $\mathrm{kHz}$; signals were filtered at $2 \mathrm{kHz}$. To isolate excitatory synaptic inputs, inhibitory synaptic inputs were totally blocked by $100 \mu \mathrm{M}$ picrotoxin. Patch pipettes (3-4 M $\Omega$ ) were borosilicate glass and filled with internal solution containing the following (in $\mathrm{mm}$ ): $9 \mathrm{KCl}, 10 \mathrm{KOH}, 120$ K-gluconate, $3.48 \mathrm{MgCl}_{2}, 10$ HEPES, $4 \mathrm{NaCl}, 4 \mathrm{Na}_{2} \mathrm{ATP}, 0.4 \mathrm{Na}_{3} \mathrm{GTP}$, and 17.5 sucrose, $\mathrm{pH} 7.25$, for testing synaptic plasticity and currentclamp (CC) recordings. The membrane potential was held at $-70 \mathrm{mV}$ in VC and CC mode. Recordings were discarded if the series resistance $\left(R_{\mathrm{s}}\right)$ varied by $>15 \%$ and the injection current for the holding potential exceeded $600 \mathrm{pA}$. PFs in the molecular layer and CFs in the granule cell layer were stimulated by an aCSF-filled electrode. We used two different protocols to induce the PF-PC LTD in CC mode for 5 min: (1) 300 times of repetitive coactivation of $\mathrm{PF}$ and $\mathrm{CF}$ at $1 \mathrm{~Hz}$ (LTD-Tetanus) and (2) 30 times of pairing-stimulation consisting of $7 \mathrm{PF}$ stimuli at $100 \mathrm{~Hz}$ followed by 1 CF stimulus delayed by $150 \mathrm{~ms}$ every $10 \mathrm{~s}$ (LTD-Burst). To evaluate PC excitability, a series of current steps of $500 \mathrm{~ms}$ duration ranging from $+100 \mathrm{pA}$ to $+500 \mathrm{pA}$ with increments of $100 \mathrm{pA}$ with a step interval of $4.5 \mathrm{~s}$ from a membrane potential of $-70 \mathrm{mV}$ was injected in CC mode.

$\mathrm{Ca}^{2+}$ imaging. $\mathrm{Ca}^{2+}$ measurements were performed with a confocal laser-scanning head (FV300, Olympus) attached to an upright confocal microscope (BX51WI, Olympus) using a $40 \times$ water-immersion objective (numerical aperture 0.8, LUMPlanFl/IR; Olympus) in parallel with electrophysiological recording. To measure dendritic $\mathrm{Ca}^{2+}$ transients, Oregon Green 488 BAPTA-1 (OGB-1, $0.2 \mathrm{~mm}$ ) was added to the internal solution in patch pipette. PCs were dialyzed for at least $30 \mathrm{~min}$ after introducing ithe ntracellular solution before imaging. Alexa Fluor 594 red fluorescence was added to saline in stimulation pipette. Confocal images were acquired with Fluoview (Olympus) software as XYT frame scans for a chosen region of interest and analyzed with ImageJ software. Baseline fluorescence frame was subtracted from maximum fluorescence frame and filtered using Gaussian blur 3D. Then, we applied the same threshold to different intensity of stimulation data and measured the area using ImageJ "Analyze particles" algorithm.

Data acquisition and analysis. All data were acquired with Clampex software (Molecular Devices) and analyzed with IgorPro 8.1 software (Wavemetrics). We evaluated the LTD by normalizing to the average of baseline PF-EPSC and recording was excluded if the baseline current or the number of evoked spikes varied by $>20 \%$. Electrical properties of the neurons were monitored with following parameters: $R_{\mathrm{S}}$ was calculated by fitting a single exponentials to the voltage responses of the test pulse $(-5 \mathrm{mV})$; input resistance $\left(R_{\mathrm{in}}\right)$ was determined from negative peak voltage deflection in response to brief hyperpolarizing current injection $(-100 \mathrm{pA} ; 100 \mathrm{~ms})$; voltage threshold $\left(V_{\text {threshold }}\right)$ of AP was defined as the voltage where the $\mathrm{dV} / \mathrm{dt}$ first exceeds $30-60 \mathrm{mV} / \mathrm{ms}$; AP amplitude was determined as difference between peak amplitude and the voltage threshold of the AP; half-width was the time duration at the halfmaximal voltage; fast afterhyperpolarization (fAHP) and medium afterhyperpolarization ( $\mathrm{mAHP}$ ) were measured by calculating the difference between voltage threshold and hyperpolarized negative peak voltage after the first AP or depolarizing square current injection, respectively. All analysis for electrical properties was performed by custom-made MATLAB code.

Data are presented as mean \pm SEM and statistical evaluations were performed using normality test, equal variant test, independent $t$ test, one-way repeated-measures (RM) ANOVA and two-way RM ANOVA with post hoc Tukey's test were performed with Origin 8.5 and SigmaPlot 12.0 software. The sample size was approved by power analysis using the $\mathrm{G}^{*}$ power 3.1.9.2.

\section{Results}

\section{LTD of intrinsic excitability of PCs accompanied by PF-PC LTD}

We first investigated whether intrinsic excitability is modulated by long-term synaptic plasticity. To observe whether excitability changes in PCs are accompanied by synaptic LTD, whole-cell PC recording was performed in 4- to 6-week-old mice in the presence of picrotoxin, a $\mathrm{GABA}_{\mathrm{A}}$ receptor inhibitor. $\mathrm{PF}-\mathrm{PC} \mathrm{LTD}$ was induced by tetanizing PF and CF simultaneously $(1 \mathrm{~Hz}, 300$ times, 5 min for the LTD-Tetanus group; Fig. 1A). The amplitude of EPSC was monitored before and after the tetanization of PF and CF to confirm the successful induction of LTD. In the same recording, intrinsic excitability was also measured in CC mode by injecting square-wised brief current step from the membrane 
A
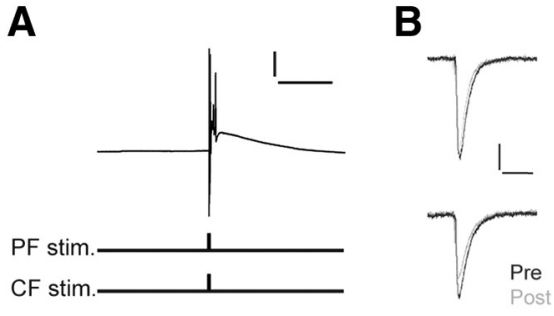

C
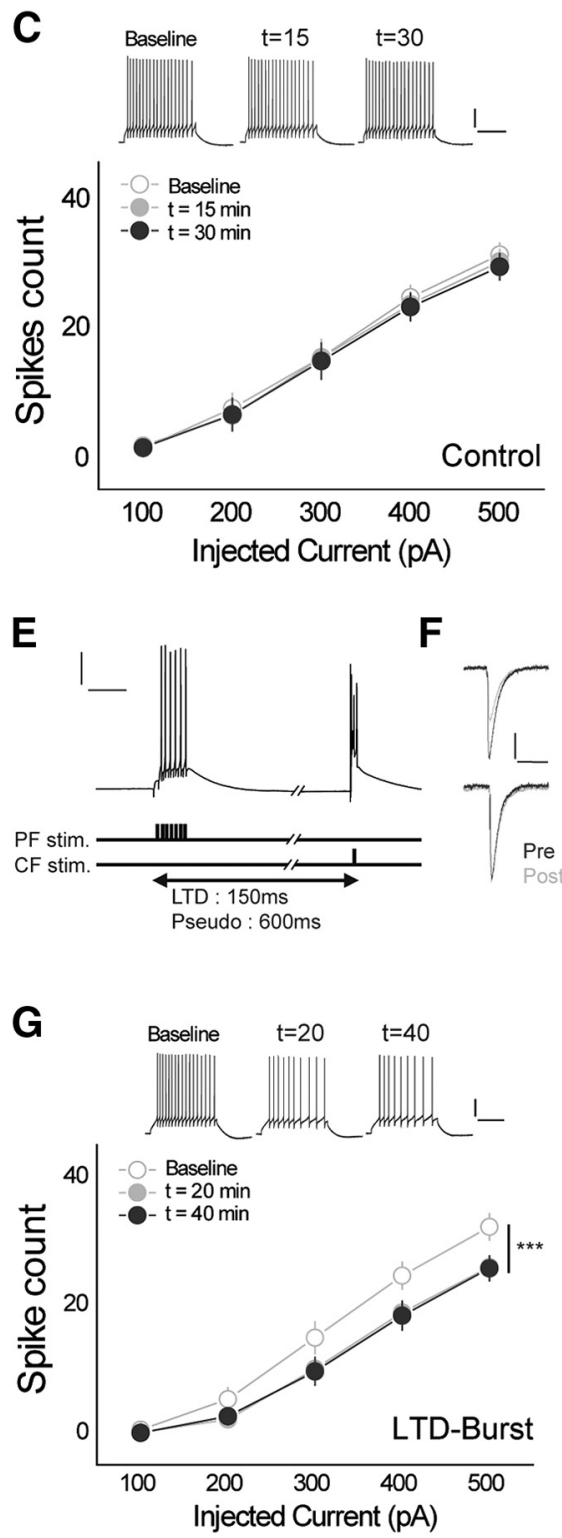
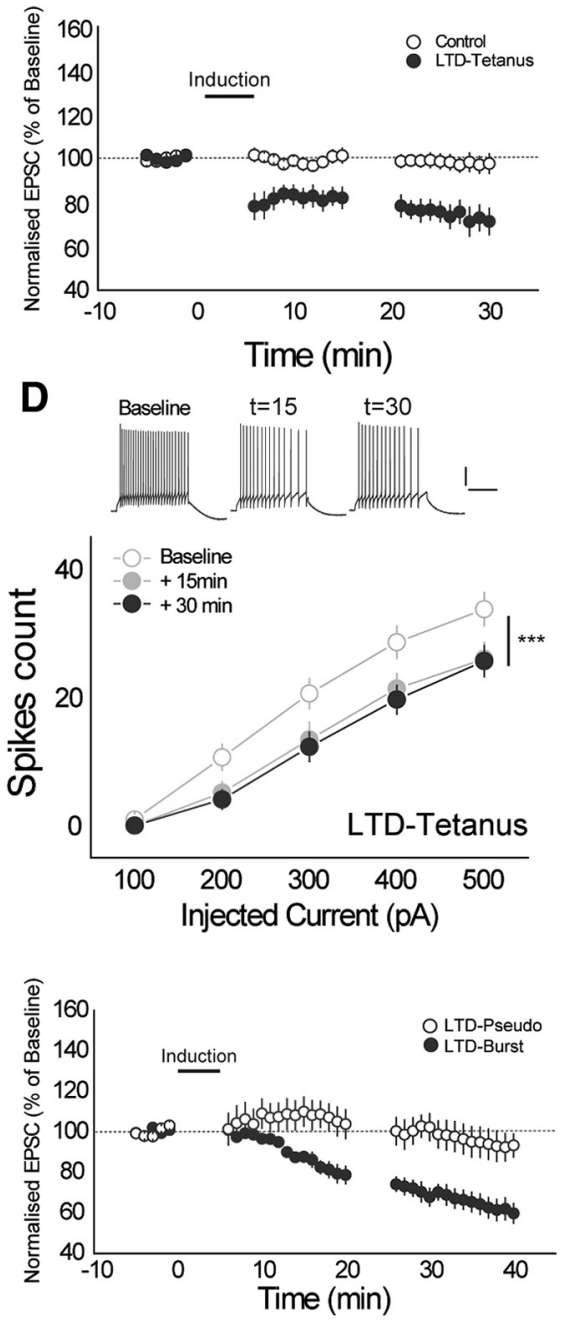

H
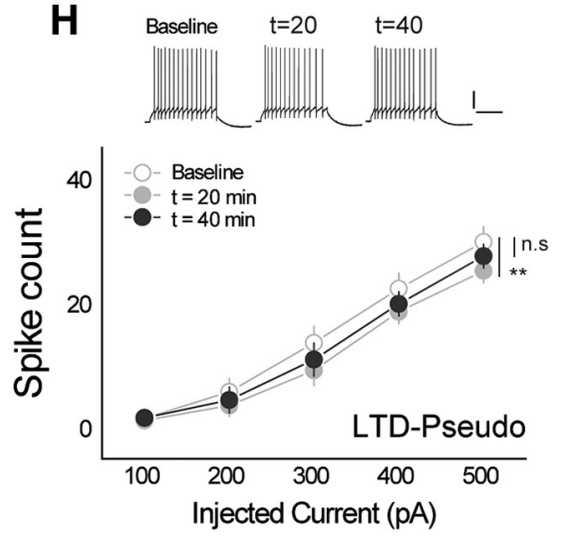

Figure 1. Synaptic LTD accompanied by LTD of intrinsic excitability. $\boldsymbol{A}, \mathrm{PF}-\mathrm{PC}$ LTD induction protocol. PF and (F were simultaneously stimulated with $1 \mathrm{~Hz}$ for $5 \mathrm{~min} 300$ times. Scale bars, $20 \mathrm{mV}$ (vertical) and $50 \mathrm{~ms}$ (horizontal). $\boldsymbol{B}$, Normalized EPSC of both groups. LTD was successfully induced in $1 \mathrm{~Hz}$ LTD group and there were no changes of EPSCs in control group. Control, open, $n=$ 9; LTD-Tetanus, filled, $n=7$. Scale bars in raw traces, $100 \mathrm{pA}$ (vertical) and $50 \mathrm{~ms}$ (horizontal). C, Intrinsic excitability of control group. Numbers of spikes were not changed during recording. Baseline, $15 \min , n=9 ; 30 \mathrm{~min}, n=8 ; F=0.219, p=0.806$. Scale bars in raw traces, $20 \mathrm{mV}$ (vertical) and $200 \mathrm{~ms}$ (horizontal). D, Intrinsic excitability of LTD-Tetanus group. Numbers of spikes were significantly reduced after LTD induction $(n=7, F=17.4, p<0.001)$. Scale bars in raw traces are the same as in $\boldsymbol{C}$. $\boldsymbol{E}$, Induction protocols for LTD-Burst and LTD-Pseudo. Thirty times of 7 PF and single CF stimulation with 150 ms interval could induce the PF-PC LTD but 600 ms interval could not. Scale bars, $20 \mathrm{mV}$ (vertical) and $50 \mathrm{~ms}$ (horizontal). F, Normalized EPSC of both groups. $150 \mathrm{~ms}$ interval protocol could successfully induce LTD and there were no changes of EPSCs in 600 ms interval protocol. LTD-Pseudo, open, $n=11$; LTD-Burst, filled, $n=10$. Scale bars in raw traces, $100 \mathrm{pA}$ (vertical) and $50 \mathrm{~ms}$ (horizontal). G, Intrinsic excitability of LTD-Burst group. Numbers of spikes were significantly reduced after LTD induction ( $F=21.1, p<0.001)$. Scale bars in raw traces, $20 \mathrm{mV}$ (vertical) and $200 \mathrm{~ms}$ (horizontal). $\boldsymbol{H}$, Intrinsic excitability of pseudo-induction group. There was statistical significance potential of $\sim-70 \mathrm{mV}$ (500 ms, from $+100 \mathrm{pA}$ to $+500 \mathrm{pA}$ with an increment of $100 \mathrm{pA}$, step interval $4.5 \mathrm{~s}$; see Materials and Methods) and determined by counting the number of depolarization-evoked spikes before and after the LTD-Tetanus protocol. Both the EPSC and the excitability were stable when tetanization was absent ( $n=9$ and $n=8$, respectably; Fig. $1 B, C)$. Simultaneous stimulation of $\mathrm{PF}$ and $\mathrm{CF}$ resulted in the EPSC reduction to $71.3 \pm 6.0 \%(n=7$; Fig. $1 B)$, which is the well established PF-PC synaptic LTD phenomenon. Concurrently, downregulation of the intrinsic excitability was accompanied by the synaptic LTD in comparison with the baseline $(n=7, F=$ 17.4, $p<0.001$, two-way RM ANOVA; Fig. $1 D$ ) in all ranges of current injection. Intrinsic excitability was shown to be significantly reduced to $76.4 \pm 4.7 \%$ of baseline when $500 \mathrm{pA}$ of current was injected after tetanization.

A previous study revealed that the PF stimulation with $1 \mathrm{~Hz}$ failed to induce intrinsic plasticity in vivo because $1 \mathrm{~Hz} \mathrm{PF}$ stimulation was not exceeding the background activity (Belmeguenai et al., 2010). To adequately reflect the activity pattern of the granule cells in vivo, we introduced the PF burst protocol (7 of $100 \mathrm{~Hz}$ PF burst followed by single CF stimulation with the stimulus interval of $150 \mathrm{~ms}, 30$ times, sweep interval $10 \mathrm{~s}, 5 \mathrm{~min}$; LTDBurst; Fig. 1E). With this protocol, synaptic LTD was also reliably elicited $(n=10$; Fig. $1 F$ ), but the synaptic plasticity was relatively slowly developed. Because of the different kinetics of decrease in EPSC, excitability was measured $20 \mathrm{~min}$ and 40 min after the induction. Consistent with the results from LTD-Tetanus protocol, the evoked spike count was considerably decreased after conjunctive activation of $\mathrm{PF}$ and CF $(n=10, F=21.1, p<0.001$, two-way RM ANOVA; Fig. $1 G$ ). A previous study described that cerebellar LTD required the precisely timed pairing of $\mathrm{PF}$ and CF activation, in which interstimuli interval was from $-50 \mathrm{~ms}$ to $+300 \mathrm{~ms}$ between PF and CF to induce synaptic plasticity (Safo and Regehr, 2008). To test whether the time window of pairing is a pivotal element for LTD-IE induction, we delivered the nonconjunctive stimulating

between baseline and the 20 min after induction $(n=11, p=$ $0.002)$, but the significance disappeared at $40 \mathrm{~min}(p=$ 0.092). Scale bars in raw traces are the same as in $C$. For statistics, two-way RM ANOVA was used for $\boldsymbol{C}, \boldsymbol{D}, \boldsymbol{G}$, and $\boldsymbol{H}$. Post hoc Tukey's test was used for different time group comparison. Error bar indicates SEM. ${ }^{* *} p<0.01,{ }^{* * *} p<0.001$. 
Table 1. Active properties of action potential before and after LTD induction

\begin{tabular}{|c|c|c|c|c|c|c|}
\hline & $V_{\text {threshold }}(\mathrm{mV})$ & AP amplitude (mV) & fAHP $(m V)$ & $\mathrm{mAHP}(\mathrm{mV})$ & FWHM (ms) & $R_{\text {in }}(\mathrm{M} \Omega)$ \\
\hline \multicolumn{7}{|l|}{ Control } \\
\hline Baseline & $-47.8 \pm 2.2$ & $69.7 \pm 6.1$ & $14.3 \pm 1.7$ & $5.1 \pm 1.2$ & $0.34 \pm 0.02$ & $83.2 \pm 6.6$ \\
\hline$t=15$ & $-47.7 \pm 1.9$ & $67.1 \pm 5.8$ & $14.5 \pm 1.6$ & $4.2 \pm 0.7$ & $0.36 \pm 0.02$ & $82.6 \pm 7.6$ \\
\hline$t=30$ & $-48.1 \pm 2.0$ & $67.7 \pm 6.2$ & $14.5 \pm 1.5$ & $3.7 \pm 0.6$ & $0.36 \pm 0.02$ & $81.4 \pm 7.4$ \\
\hline \multicolumn{7}{|l|}{ LTD-Tetanus } \\
\hline Baseline & $-49.3 \pm 1.0$ & $86.6 \pm 4.2$ & $13.5 \pm 0.7$ & $7.4 \pm 0.6$ & $0.33 \pm 0.02$ & $78.0 \pm 4.2$ \\
\hline$t=15$ & $-50.9 \pm 1.4$ & $85.0 \pm 3.4$ & $14.1 \pm 0.5$ & $4.8 \pm 0.3^{* * *}$ & $0.33 \pm 0.02$ & $70.4 \pm 3.5^{*}$ \\
\hline$t=30$ & $-52.3 \pm 1.8^{*}$ & $85.8 \pm 3.7$ & $13.4 \pm 0.6$ & $4.0 \pm 0.6^{* * *}$ & $0.33 \pm 0.02$ & $66.4 \pm 3.4^{* * *}$ \\
\hline \multicolumn{7}{|l|}{ LTD-Pseudo } \\
\hline Baseline & $-48.8 \pm 1.2$ & $74.0 \pm 2.2$ & $12.4 \pm 0.6$ & $6.7 \pm 0.6$ & $0.31 \pm 0.01$ & $70.2 \pm 3.9$ \\
\hline$t=20$ & $-49.2 \pm 1.2$ & $74.3 \pm 1.9$ & $12.6 \pm 0.7$ & $4.8 \pm 0.6^{* * *}$ & $0.32 \pm 0.00$ & $66.4 \pm 3.9^{*}$ \\
\hline$t=40$ & $-49.2 \pm 1.3$ & $73.6 \pm 2.2$ & $13.2 \pm 0.7$ & $4.0 \pm 0.4^{* * *}$ & $0.32 \pm 0.01$ & $67.1 \pm 4.0$ \\
\hline \multicolumn{7}{|l|}{ LTD-Burst } \\
\hline Baseline & $-47.7 \pm 0.9$ & $81.4 \pm 3.9$ & $13.1 \pm 0.7$ & $7.3 \pm 0.6$ & $0.28 \pm 0.01$ & $69.5 \pm 4.0$ \\
\hline$t=20$ & $-48.7 \pm 0.7$ & $81.1 \pm 4.0$ & $14.0 \pm 0.5$ & $5.0 \pm 0.4^{* * *}$ & $0.29 \pm 0.00$ & $62.6 \pm 4.4^{* *}$ \\
\hline$t=40$ & $-48.7 \pm 1.0$ & $79.7 \pm 4.3$ & $13.5 \pm 0.5$ & $4.5 \pm 0.6^{* * *}$ & $0.30 \pm 0.01$ & $63.8 \pm 3.3^{*}$ \\
\hline
\end{tabular}

Among active membrane properties, voltage threshold $\left(V_{\text {threshold }}\right)$ was significantly changed in the LTD-Tetanus group ( 30 min, $\left.p=0.012\right)$. Medium afterhyperpolarization (mAHP) was considerably decreased in all stimulated groups, including the LTD-Tetanus, LTD-Pseudo, and LTD-Burst groups (all groups, $p<0.001$ ). Other parameters, such as AP amplitude, fast AHP (fAHP), and full-width-half-maximum (FWHM), were not altered. Asterisks in here marked by post hoc Tukey's test after one-way RM ANOVA. ${ }^{*} p<0.05,{ }^{* *} p<0.01,{ }^{* * *} p<0.001$.

(LTD-Pseudo) protocol (Fig. 1E). In an LTD-Pseudo protocol delivered condition, synaptic depression was not induced $(n=$ 11; Fig. $1 F$ ). Interestingly, intrinsic excitability was decreased 20 min after induction; however, the reduction was recovered 40 min after application of LTD-Pseudo $(t=20, p=0.002 ; t=40$, $p=0.092$; post hoc Tukey's test after two-way RM ANOVA; Fig. $1 H)$. These results indicated that uncoupled conjunction of the $\mathrm{PF}$ and CF was insufficient for the maintenance of the intrinsic plasticity.

To further examine changes in the intrinsic properties, we analyzed the properties of action potential (AP) (Table 1). We found that the voltage threshold was shifted to $\sim 3 \mathrm{mV}$ of hyperpolarization $30 \mathrm{~min}$ after the tetanization $(n=7,-49.3 \pm 1.0$ (baseline) vs $-52.3 \pm 1.8(t=30), p=0.012$, post hoc Tukey's test after one-way RM ANOVA), but there were no changes in other groups. The reduction of medium after-hyperpolarization (mAHP) was observed in all groups except the control group. These reductions were most obvious and significant among the properties of AP [LTD-Tetanus; $7.4 \pm 0.6 \mathrm{mV}$ (baseline) vs $3.8 \pm$ $0.6 \mathrm{mV}(t=30), F=28.803, p<0.001$, LTD-Burst; $7.3 \pm 0.6$ (baseline) vs $4.5 \pm 0.6(t=40), F=24.3, p<0.001$, LTD-Pseudo; $6.7 \pm 0.6$ (baseline) vs $4.0 \pm 0.4(t=40), F=28.4, p<0.001$, one-way RM ANOVA]. Robust reduction of $R_{\text {in }}$ was also observed in LTD-induced neurons (LTD-Tetanus; $85.7 \pm 3.0 \%$, $p<0.001$, LTD-Burst; $92.2 \pm 1.9 \%, p=0.023$, post hoc Tukey's test after one-way RM ANOVA; Table 1). The change of $\mathrm{R}_{\text {in }}$ in LTD-Pseudo group showed similar pattern to that of the excitability. It was slightly reduced $20 \mathrm{~min}$ after induction, but recovered in $40 \mathrm{~min}(t=20,94.6 \pm 2.1 \%, p=0.027 ; t=40,95.7 \pm$ $2.3 \%, p=0.082$, post hoc Tukey's test after one-way RM ANOVA). The changes of the other parameters were less prominent. Given the altered AP properties, LTD-IE was derived from altered intrinsic properties and did not result from the decreased synaptic weight.

\section{LTD-IE has different developing kinetics from synaptic LTD}

We observed an excitability change accompanied with PF-PC LTD. Because the excitability change was monitored at two time points after LTD induction, the kinetics of the excitability change was not shown. To clarify this, the depolarizing current eliciting $10-20$ spikes (100 to $300 \mathrm{pA}$ of current) was injected and the spike count was measured continuously in the CC mode before

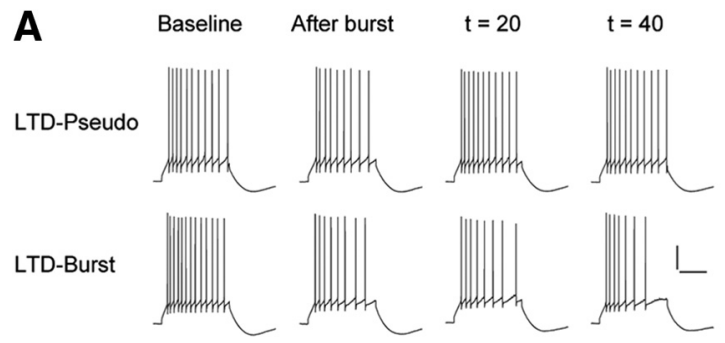

B

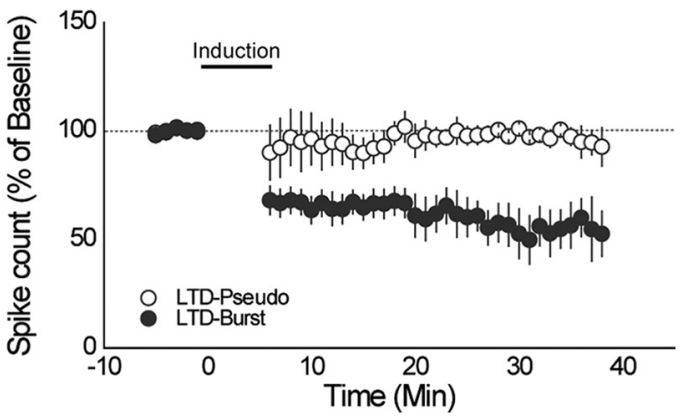

Figure 2. LTD-IE showed an immediate reduction after induction. $\boldsymbol{A}$, Raw traces in several time points. Scale bars, $20 \mathrm{mV}$ (vertical) and $200 \mathrm{~ms}$ (horizontal). $\boldsymbol{B}$, Normalized spike count. LTD-Pseudo group showed a slight reduction after stimulation, but its spike count gradually rises and was fully recovered at 20 min (open, $n=7$ ). Unlike the LTD-Pseudo group, the LTD-Burst group showed an immediate reduction after the induction protocol (filled, $n=10$ ).

and after induction protocols. We found that excitability decreased immediately after LTD induction and the magnitude of intrinsic plasticity showed slight further development, but did not reach the statistical significance (Fig. $2 A, B$ ). When we applied LTD-Pseudo protocol as a control, there were no significant changes in spike count (Fig. 2A,B).

\section{Number of recruited synapses was not correlated to the magnitude of the neuronal plasticity}

LTD-IE is the concomitant of paired stimulation of PF with CF and incomplete paired stimuli could not lead to persistent excitability change. This observation indicates that the coincidence of synaptic activation is a prerequisite for the intrinsic plasticity. We 

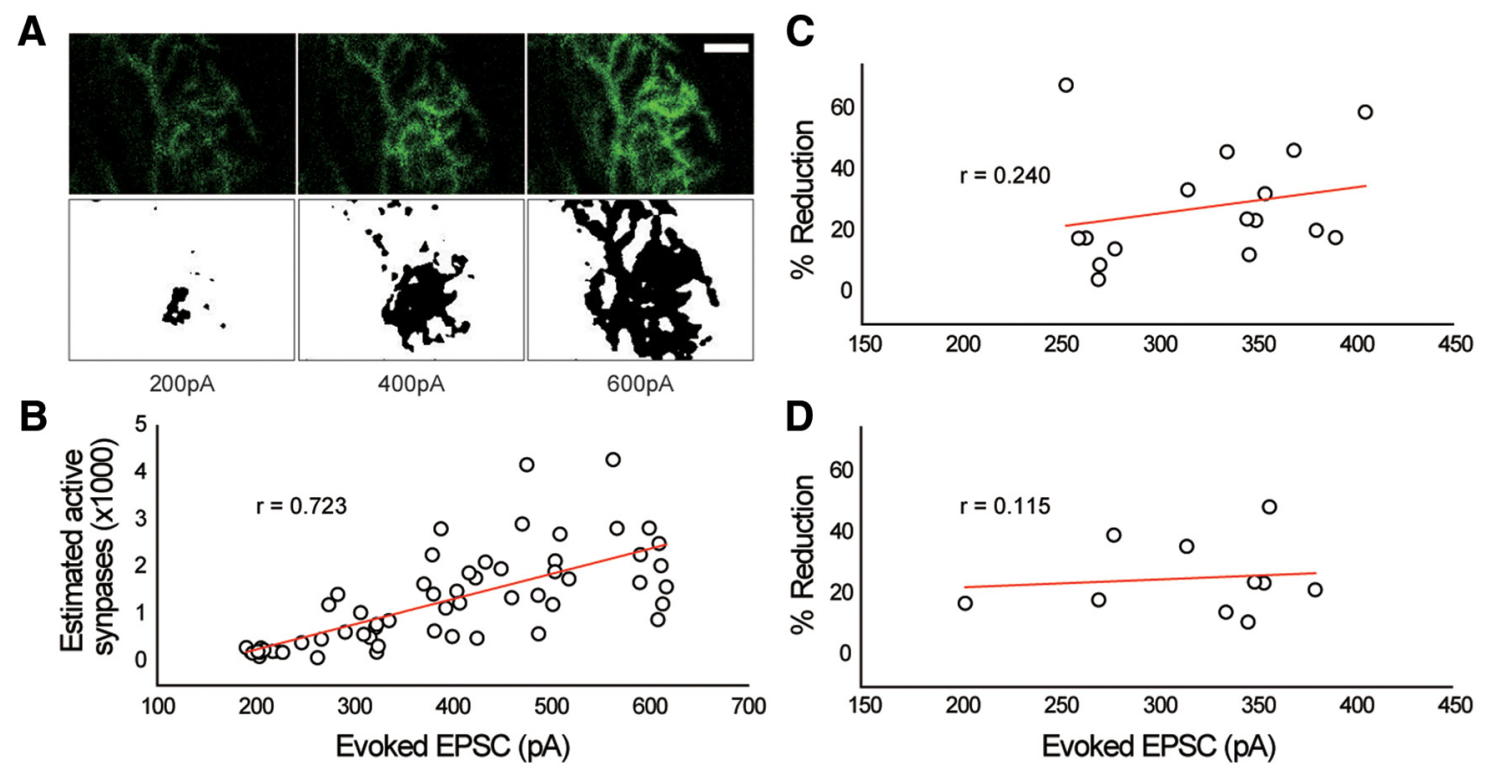

Figure 3. Correlation between recruited number of synapses and plasticity. $\boldsymbol{A}$, Confocal $\mathrm{Ca}^{2+}$ images (top) and their binary images (bottom) for analysis. The activated dendritic area was increased by an increment of stimulation intensity. Scale bars, $10 \mu \mathrm{m}$. $\boldsymbol{B}$, Linear regression between estimated the number of active synapses and stimulation intensity. There was a positive correlation between the number of active synapses and stimulation intensity (Pearson's $r=0.723, p<0.001$ ). C, Correlation between reduced excitability in LTD-IE and stimulation intensity. There was no correlation between reduced excitability and stimulation intensity (Pearson's $r=0.240, p=0.370$ ). $D$, Correlation between the magnitude of synaptic LTD and stimulation intensity. There was no correlation between magnitude of synaptic LTD and stimulation intensity (Pearson's $r=0.115, p=0.752$ ).

thus investigated whether LTD-IE is dependent on the number of activated synapses by synaptic stimulation. Given that a previous study described the number of synapses on PC dendrite by its area through electron microscopy measurement (Napper and Harvey, 1988), confocal $\mathrm{Ca}^{2+}$ imaging was performed to quantify the extent of the activated dendritic area in response to PF stimulation and estimated the number of synapses recruited by LTD induction. The stimulation intensity was adjusted to elicit from $\sim 200$ to $600 \mathrm{pA}$ of the evoked EPSC. With this intensity, we delivered LTD-Burst protocol without CF stimulation and estimated the number of recruited synapses based on the previous report. In this recording, we confirmed that larger stimuli intensity caused activation of more synapses $(p<0.001, r=0.72$; Fig. $3 A, B)$. Based on this result, we analyzed the correlation between stimulation intensity and magnitude of the LTD-IE. The magnitude of the excitability change was not stimulation intensity dependent ( $p=0.37, r=0.24$; Fig. $3 C$ ), indicating that there was no correlation between the numbers of activated synapses and intrinsic plasticity. In addition, we found that synaptic LTD also did not show significant correlation with stimulation intensity $(p=$ $0.75, r=0.11$; Fig. $3 D$ ). Temporal coincidence of PF and CF activation is the more significant element for the induction of the PC plasticity than the number of recruited active synapses.

\section{LTD-IE was not reversed by subsequent LTP-IE induction}

Synaptic LTP and LTD are complementary. Indeed, reversible changes in synaptic efficacy at PF-PC synapses has been shown in in vitro slice recording and behavior tests (Boyden and Raymond, 2003; Coesmans et al., 2004). These findings lead to the question of whether intrinsic plasticity also shows this reversibility. To test this, we developed the LTP-IE protocol modified from previous studies (Belmeguenai et al., 2010; Nataraj et al., 2010). A precisely timed pulse train $(5 \mathrm{~Hz} / 3 \mathrm{~s}$, pulse duration: $100 \mathrm{~ms}$ ) was injected every $5 \mathrm{~s}$ for $5 \mathrm{~min}$ in CC mode (Fig. $4 A$, top). The amplitude of pulse train was 100-150 pA higher than injecting current eliciting 5-15 spikes. Application of this protocol exhibited the potentia- tion of spike count ( $n=9, F=6.70, p<0.001$, one-way RM ANOVA; Fig. 4A).

After confirmation of the LTP-IE protocol, we delivered two types of serial-ordered protocol as follows: (1) LTP-IE-LTP-IELTD-Burst-LTP-IE (PPDP serial) and (2) LTD-Burst-LTDBurst-LTP-IE-LTD-Burst (DDPD serial). In the PPDP serial, the first application of LTP-IE significantly potentiated PC excitability (135.2 $\pm 3.3 \%$ of baseline, $n=10, p=0.043$, post hoc Tukey's test after one-way RM ANOVA compared with the value of the prior period; Fig. $4 B$ ), but there were no further changes after the second induction of LTP-IE (144.5 $\pm 8.5 \%$ of baseline, $p=0.939)$. Application of the LTD-Burst protocol and the third LTP protocol resulted in slight depotentiation $(123.1 \pm 14.9 \%$ of baseline) and repotentiation of excitability (141.9 $\pm 18.1 \%$ of baseline), respectively, but these changes were not significantly different from that measured before induction $(p=0.404$ and $p=0.654$, respectively). Next, we applied DDPD serial to test whether the LTP-IE protocol reversed LTD-IE. LTD-IE was fully saturated immediately after application of the LTD-inducing protocol (42.5 $\pm 5.3 \%$ of baseline, $n=4, p<0.001$, post hoc Tukey's test after one-way RM ANOVA compared with the value of prior period; Fig. $4 C$ ); therefore, further reduction of excitability was not observed after the second induction of LTD (36.5 \pm $6.1 \%$ of baseline, $p=0.847$ ). Application of the LTP-IE protocol could not result in a significant change of the excitability compared with that measured after the first and second induction of LTD $(41.0 \pm 10.6 \%$ of baseline, $p=0.940)$. Finally, the third LTD-Burst protocol was delivered and there was no alteration of spike count $(38.3 \pm 8.8 \%$ of baseline, $p=0.991)$. These data suggested that the intrinsic plasticity of PCs cannot be reversed by the opposite direction of plasticity induction.

\section{LTD-IE required $\mathrm{Ca}^{2+}$ signal but depended on}

$\mathrm{Ca}^{2+}$-activated $\mathrm{K}^{+}$channels

What is the intracellular signaling involved in the intrinsic plasticity? As we described above, precisely paired activation of PF 
A

A
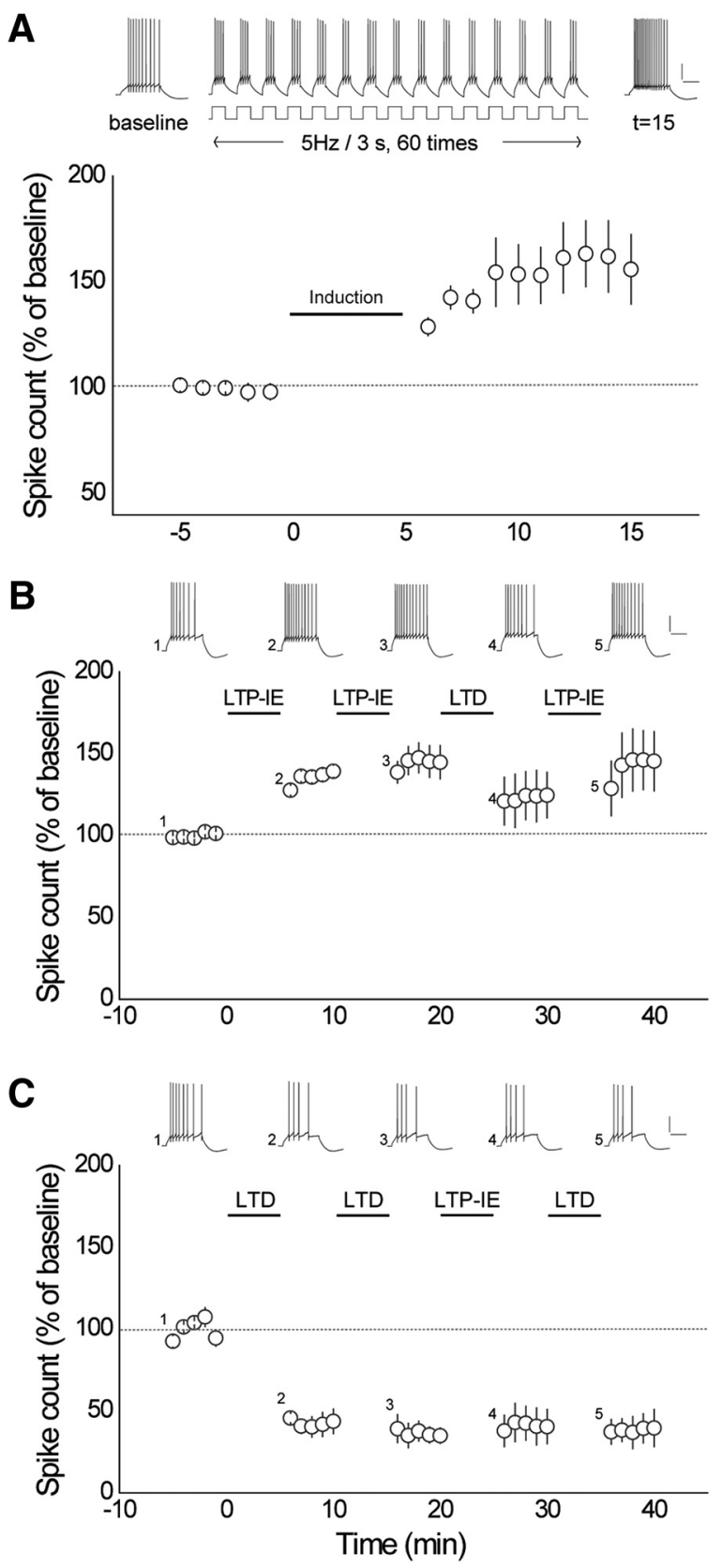

Figure 4. Reverse of intrinsic plasticity. A, LTP-IE was induced by induction protocol. Raw traces in baseline, induction protocol, and $15 \mathrm{~min}$ after induction (top, left to right). Excitability was directly increased and maintained after induction (bottom, $n=9, p<0.001$ ). $\boldsymbol{B}$, Normalized spike count under the PPDP serial. The first LTP-IE stably induced potentiation of excitability $(n=10, p=0.043)$ and there was no further potentiation after the second LTP-IE induction ( $p=0.939$, cf. prior period). After LTD, the third LTP induction brought slight depotentiation and repotentiation, respectively, but this was not significant (LTD induction, $p=0.404$; the third LTP induction, $p=0.654$, cf. prior period). C, Normalized spike count under DDPD serial. Like the PPDP serial, the first LTD induced reduction of excitability $(n=4, p<0.001)$ and no further changes after the second LTD induction ( $p=0.847$ ). LTP-IE induction and the third LTD had no effect on excitability (LTP-IE induction, $p=940$; the third LTD induction, $p=0.991$ ). For statistics, one-way RM ANOVA was used. Post hoc Tukey's test was used for different time group comparisons. Error bar indicates SEM.

and $\mathrm{CF}$ is a pivotal factor for the induction of both synaptic and intrinsic plasticity. This suggests that the LTD-IE may share a signaling pathway with PF-LTD. Therefore, we first investigated whether the LTD-IE is dependent on $\mathrm{Ca}^{2+}$ signaling, which has been implicated in determining the PF-PC LTD (Konnerth et al.,
1992). When intracellular $\mathrm{Ca}^{2+}$ signaling was blocked by adding the $\mathrm{Ca}^{2+}$ chelator BAPTA $(10 \mathrm{~mm})$ to the pipette solution, PF-PC LTD was completely abolished ( $n=10$; Fig. $5 A)$. In the same recording, prominent change of excitability after LTD induction was not exhibited in presence of BAPTA $(n=5, F=$ $1.262, p=0.334$; Fig. $5 B$ ), suggesting that $\mathrm{Ca}^{2+}$ is necessary for the intrinsic plasticity. Intracellular $\mathrm{Ca}^{2+}$ derived from synaptic activation governs a variety of $\mathrm{Ca}^{2+}$-sensing molecules, such as PKC. To investigate whether LTD-IE requires PKC activation as in synaptic plasticity, the LTD-Burst protocol was delivered with an internal solution including the PKC inhibitor peptide PKC(19-36). Interestingly, although both synaptic and intrinsic plasticity were normally induced and maintained in the presence of inactive control peptide, [Glu $\left.{ }^{27}\right]$-PKC (19-36) (synaptic plasticity: $n=7$; intrinsic plasticity: $n=8, F=18.815, p<0.001$, one-way RM ANOVA; Fig. 5C,D), changes of synaptic efficacy and excitability were not induced by $\mathrm{PKC}(19-36)$ (synaptic plasticity: $n=6$; intrinsic plasticity: $n=13, F=1.618, p=0.211$, one-way RM ANOVA). The LTD-IE might share a common intracellular pathway with PF-LTD because PKC activation underlies LTD-IE.

It has been reported widely that the intrinsic plasticity is derived from the changes in ion channel conductance and/or expression (Belmeguenai et al., 2010; Narayanan et al., 2010; van Welie and du Lac, 2011; Shim et al., 2016). We observed that mAHP was significantly reduced whereas fAHP was unchanged by the application of LTD-inducing protocols. It has also been reported widely that the small conductance $\mathrm{Ca}^{2+}$-activated $\mathrm{K}^{+}$ (SK) channels determine the mAHP rather than fAHP. In addition, SK channels have been regarded as the major component for LTP-IE (Belmeguenai et al., 2010; Grasselli et al., 2016). Therefore, we investigated whether this type of ion channel was required for the excitability change. Bath application of apamin (100 nM), a selective antagonist of SK channels, did not affect to the induction of synaptic and intrinsic plasticity (synaptic plasticity: $n=7$; intrinsic plasticity: $n=7, F=11.317, p<0.001$, one-way RM ANOVA; Fig. $5 E, F)$. Therefore, we conclude that activity-dependent downregulation of intrinsic excitability is not derived from $\mathrm{Ca}^{2+}$-activated $\mathrm{K}^{+}$channel, but rather the $\mathrm{Ca}^{2+}$ dependency of LTD-IE.

\section{Information processing after LTD induction}

Given that cerebellar PCs integrate tremendous input from the subcortical region and generate the final output signal to its relay neurons, we questioned whether the strategy for the information processing would be changed during the alteration of intrinsic excitability after synaptic depression. To answer this, two types of information processing in PCs were investigated: (1) temporally distributed input signal integrated within a dendrite (Fig. $6 \mathrm{~A}$ ) and (2) spike frequency adaption (SFA), which operates to filter the output signal by attenuating unnecessary firing (Benda and Herz, 2003; Pozzorini et al., 2013; Fig. 6D). Temporal summation was evaluated by calculating the ratio of fifth EPSP to the first elicited by $5 \mathrm{PF}$ stimuli with $10 \mathrm{~Hz}$. Reduction of EPSP was observed 40 min after LTD-Burst but not LTD-Pseudo compared with baseline $(t=40$, LTD-Pseudo, $n=11,1.1 \pm 1.0$; LTD-Burst, $n=9$, $0.7 \pm 1.1 ; p=0.019$, post hoc Tukey's test after two-way RM ANOVA; Fig. 6B). The EPSP5/EPSP1 ratio, nonetheless, was not changed $[t=40,0.9 \pm 0.06$ (LTD-Pseudo) vs $0.9 \pm 0.04$ (LTDBurst), $p=0.804$, independent $t$ test; Fig. $6 C$ ], indicating that the PF-LTD resulted in a decrease of the absolute EPSP value without changes in the strategy for the temporal summation of the inputs. 

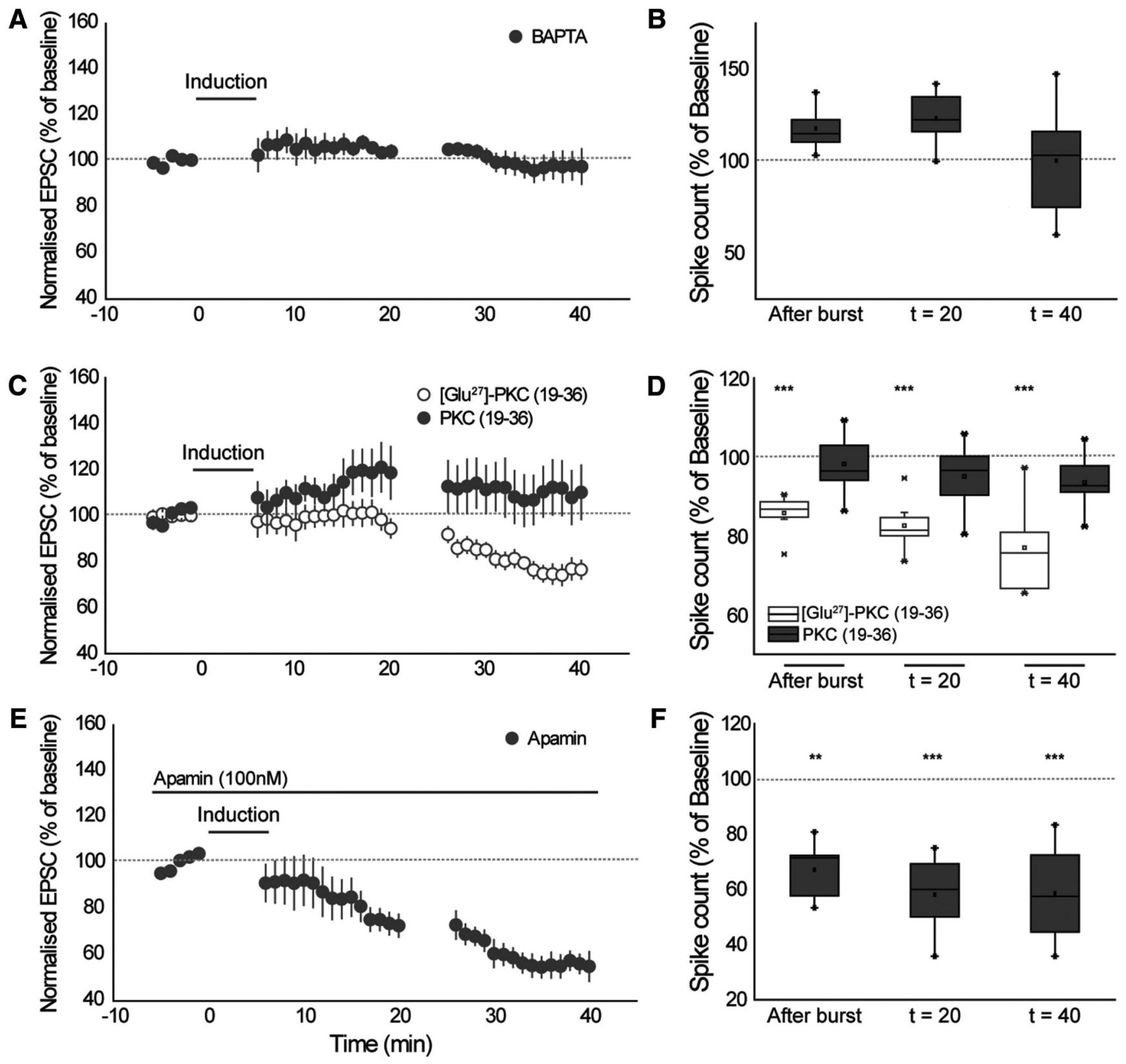

Figure 5. Synaptic LTD and LTD-IE share the intracellular $\mathrm{Ca}^{2+}$ and PKC pathway as an underlying mechanism but SK channels were not related. $A, B$, LTD induction under BAPTA-containing internal solution. $\boldsymbol{A}$, PF-PC LTD was completely blocked by $10 \mathrm{~mm}$ BAPTA $(n=10) \cdot \boldsymbol{B}$, LTD-IE was also abolished under high concentration of $B A P T A(n=5, F=1.703, p=0.219)$. $\boldsymbol{C}, \boldsymbol{D}$, PKC inhibitor and control peptide treatment. C, PKC inhibitor peptide, PKC (19-36) (10 $\mu \mathrm{m})$, blocked LTD induction (filled, $n=6)$, but LTD was successfully induced in control peptide ([Glu $\left.{ }^{27}\right]$ PKC(19-36), 10 $\mu \mathrm{M} ;$ open, $n=7$ ). $\boldsymbol{D}$, Excitability of control peptide group showed LTD-IE from immediately after induction protocol to end of the recording (after burst, $n=8 ; 20 \mathrm{~min}, n=8 ; 40 \mathrm{~min}, n=6 ; F=$ $13.754, p<0.001$ ), but no significant reduction of excitability was observed in PKC inhibitor group (after burst, $n=13 ; 20 \mathrm{~min}, n=8 ; 40 \mathrm{~min}, n=6 ;(F=1.618, p=0.211) . \boldsymbol{E}, \boldsymbol{F}, \mathrm{SK}$ channel blocker treatment. $\boldsymbol{E}$, Apamin $(100 \mathrm{~nm})$ was not able to block LTD induction $(n=7)$. $\boldsymbol{F}$, LTD-IE was induced. Excitability of each time point was significantly reduced (after burst, $n=5 ; 20 \mathrm{~min}, n=$ $5 ; 40 \min , n=4 ; F=16.255, p<0.001)$. For statistics, one-way RM ANOVA was used for $\boldsymbol{B}, \boldsymbol{D}$, and $\boldsymbol{F}$. Post hoc Tukey's test was used for different time group comparisons. Error bar indicates SEM. ${ }^{* *} p<0.01,{ }^{* * *} p<0.001$.

To determine the SFA, all interspike intervals (ISIs) of the evoked spike train elicited by injecting current of $500 \mathrm{pA}$ were calculated and normalized to the first ISIs (Fig. 6E). Despite the reduction of the firing rates corresponding to the synaptic LTD, there was no significant change in calculated SFA compared with the baseline in all groups regardless of what protocol was used (Fig. $6 F-I$ ). We concluded that synaptic LTD might not manifest the changes in the strategy of information process, but rather modify gain responses for generating the AP spike.
LTD of flocculus PCs and consequent induction of LTD-IE

The flocculus, a part of the cerebellum, is thought of as the core region for VOR learning and synaptic plasticity in this area accordingly plays a pivotal role in the behavior (Ito, 1982). Because a recent study confirmed the presence of PF-PC LTD in flocculus (Suvrathan et al., 2016), we investigated whether LTD-IE also is able to be triggered by synaptic LTD in the flocculus. First, we reproduced synaptic LTD in the flocculus with the LTD-Burst protocol, because it satisfies the critical time window mentioned 
A

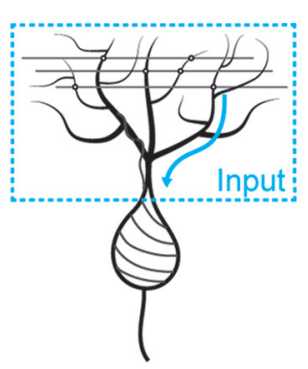

D

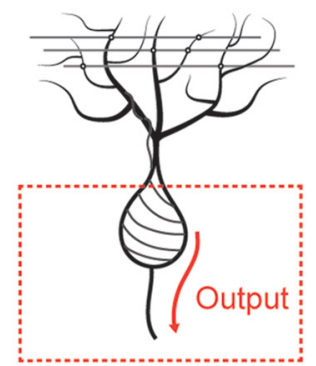

E

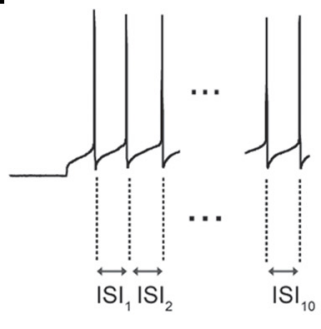

B

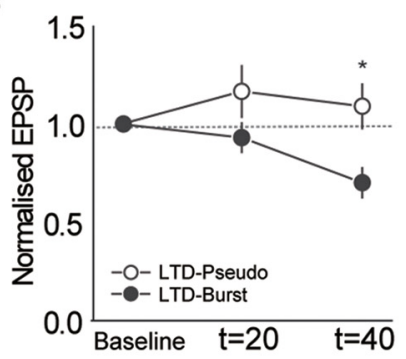

F

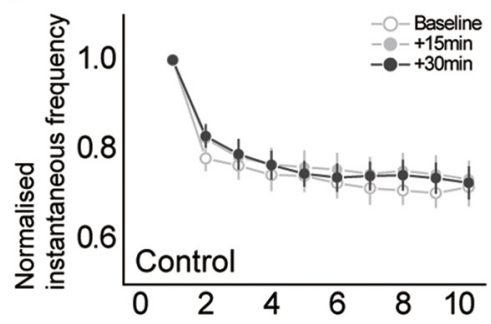

H

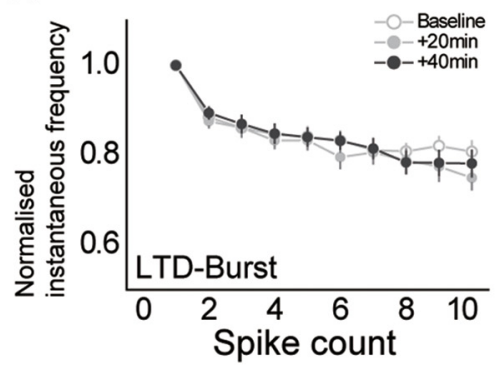

C

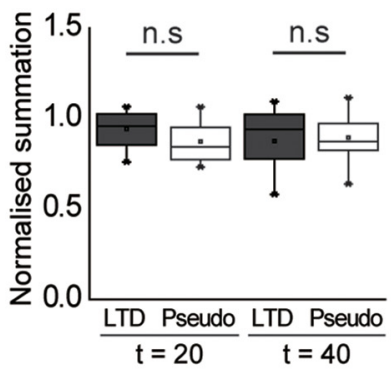

G

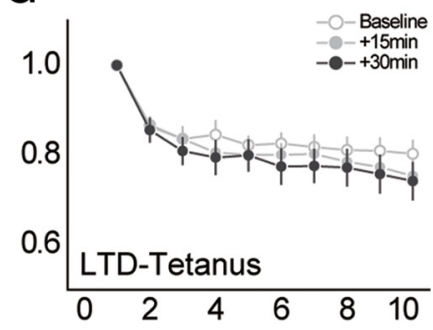

I

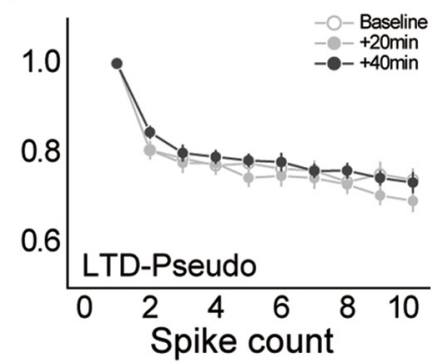

Figure 6. Information processing strategy was not changed after LTD-IE. $\boldsymbol{A}$, Schematic for input processing in PCs. $\boldsymbol{B}$, Normalized EPSP in LTD-Pseudo and LTD-Burst groups. PF-LTD caused reduction of EPSP compared with the LTD-Pseudo group (LTD-Pseudo, $n=11 ;$ LTD-Burst; $n=9, F=4.6, p=0.048$ ). C, Normalized EPSP summation in LTD-Pseudo and LTD-Burst groups. The normalized summation value was not influenced by LTD induction. (LTD-Pseudo, $n=11$; LTD-Burst; $n=9 ; 20$ min, $p=0.167 ; 40$ min, $p=0.084$ ). $D$, Schematic for output processing in PCs. $\boldsymbol{E}$, Counting ISIs. Ten ISIs were measured to analyze SFA. $\boldsymbol{F}-\boldsymbol{I}$, Normalized instantaneous frequency of all groups. Through time points, the power of frequency adaptation was not changed in the control $(\boldsymbol{F})$, LTD-Tetanus $(\boldsymbol{G})$, LTD-Burst $(\boldsymbol{H})$, and LTD-Pseudo ( $\boldsymbol{I}$ ) groups. For statistics, two-way RM ANOVA was used for $\boldsymbol{B}$ and independent $t$ test for $\boldsymbol{C}$. Post hoc Tukey's test was used for different time group comparisons. Error bar indicates SEM. ${ }^{*} p<0.05$.

in Suvrathan et al. (2016). In this recording, a robust decrease in EPSC amplitude was observed after LTD induction ( $57.5 \pm 8.0 \%$, $n=9$; Fig. 7A) and the depressed values were similar to the LTD-induced PCs in the vermis. In the same recording, we measured the excitability of PCs 20 and $40 \mathrm{~min}$ after the induction. LTD-IE of floccular PCs indeed was reliably triggered by LTD induction $(n=8, F=12.9, p<0.001$, two-way RM ANOVA; Fig. $7 B)$. Furthermore, the LTD in the vermis, $R_{\mathrm{in}}$, was significantly reduced after burst induction protocol $(t=40,78.3 \pm 5.0 \%, p<$ 0.001 , post hoc Tukey's test after one-way RM ANOVA; Table 2) and a decrease of mAHP was also seen [8.7 \pm 0.9 (baseline) vs $4.4 \pm 1.1(t=40), F=42.5, p<0.001$, one-way RM ANOVA]. The voltage threshold was hyperpolarized $40 \mathrm{~min}$ after induction, which is in consistent with the experiment from the vermis $[-47.6 \pm 1.2$ (baseline) vs $-49.5 \pm 1.2(t=40), p=0.011$, post hoc Tukey's test after one-way RM ANOVA; Table 2]. These results indicate that the PCs in the flocculus change their spiking activity when synaptic plasticity occurs and the direction of the activity change is similar to that in the vermis.

Next, to test whether the LTD-IE would contribute to dendritic and somatic information processing in the flocculus, we monitored temporal EPSP summation and analyzed the SFA. The amplitude of the EPSP was decreased by application of the
LTD-Burst protocol $(t=40, n=8,64.0 \pm 0.1 \%, p=0.005$, post hoc Tukey's test after one-way RM ANOVA; Fig. 7C). The summation ratio of the EPSP in response to $5 \mathrm{PF}$ stimuli showed no significant changes in parallel with the results shown in vermis $[1.1 \pm 0.1(t=20)$ vs $1.0 \pm 0.1(t=40), p=0.428$, independent $t$ test; Fig. $7 D$ ]. Furthermore, none of the change was shown in SFA, when the instantaneous firing frequency was normalized to the first firing frequency (Fig. $7 E$ ). Therefore, as in the vermis, temporal summation and SFA did not sensitively reflect the synaptic and intrinsic plasticity in the flocculus. These results indicate that output signal of learned PCs might derive from reducing the absolute value of the signal and does not depend on the patterns of information processing.

\section{Discussion}

In this study, we show that conjunctive activation of PF and CF that induces of PF-PC LTD downregulate the intrinsic excitability, resulting in the synergistic decrease of neuronal net output. This novel phenomenon shares intracellular $\mathrm{Ca}^{2+}$ signaling and PKC pathway with synaptic LTD, but is not regulated by SK channels, which were known as critical factors for LTP-IE. In information processing, dendritic temporal summation and SFA power were not changed by the LTD-IE, indicating that the strat- 
A

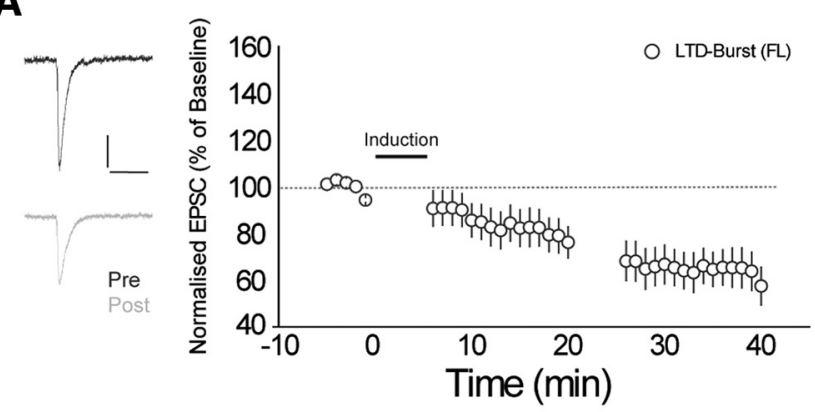

B
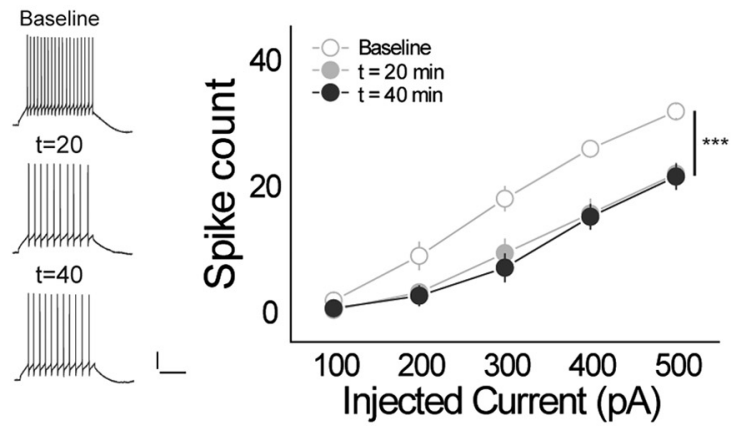

C

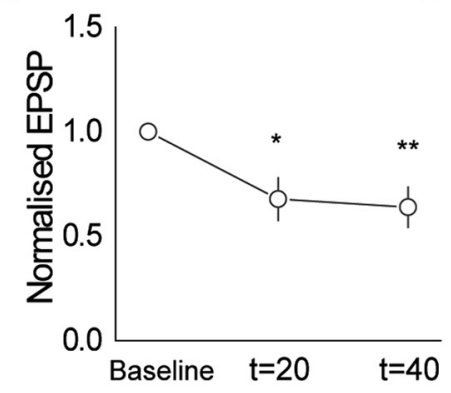

E

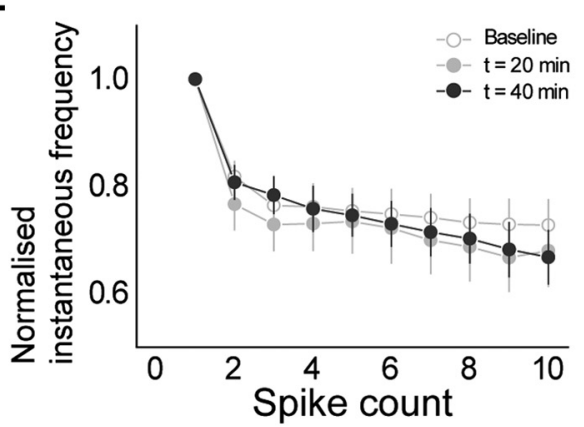

Figure 7. LTD and LTD-IE in the cerebellar flocculus. $A$, Normalized EPSC in flocculus. PF-PC LTD was successfully induced in flocculus $(n=8)$. Scale bars in raw traces, $100 \mathrm{pA}$ (vertical) and $50 \mathrm{~ms}$ (horizontal). $\boldsymbol{B}$, Intrinsic excitability of LTD-Burst group. Numbers of spikes were significantly reduced after LTD induction $(F=12.9, p<0.001)$. Scale bars in raw traces, $20 \mathrm{mV}$ (vertical) and $200 \mathrm{~ms}$ (horizontal). $C$, Normalized EPSP. PF-LTD causes reduction of EPSP $(F=8.9, p=0.003 ; 20 \mathrm{~min}, p=0.011 ; 40 \mathrm{~min}, p=0.005)$. $D$, Normalized EPSP summation. There was no statistical significance between the two time points $(p=0.428)$. $\boldsymbol{E}$, Normalized instantaneous frequency in the flocculus group. The power of frequency adaptation was not changed before and after LTD induction. For statistics, two-way RM ANOVA was used for $\boldsymbol{B}$, one-way RM ANOVA for $\boldsymbol{C}$ and $\boldsymbol{D}$, and paired $t$ test for $\boldsymbol{E}$. Post hoc Tukey's test was used for different time group comparisons. Error bar indicates SEM. ${ }^{*} p<0.05,{ }^{* *} p<0.01,{ }^{* * *} p<0.001$. egy for the handling information of PCs insensitively reflects the LTD induction.

Bidirectional modulation of the intrinsic excitability in PCs Intrinsic plasticity complements the synaptic transmission and enables fulfillment of optimal signal transduction within a local circuit in a region- and cell-type-specific manner (Brager and Johnston, 2007; Nataraj et al., 2010; Mahon and Charpier, 2012). In the cerebellum, there are two possible expectations in terms of the direction of intrinsic plasticity associated with synaptic LTD. First, PF-PC LTD may induce reduction of the intrinsic excitability of PCs, leading to disinhibition on the next relay neurons such as neurons in the DCN or the VN. In this case, PC excitability is modulated with the same direction of PF-PC synaptic modification, resulting in enhanced activity of the DCN and the VN via disinhibition. Alternatively, PF-PC LTD may induce LTP-IE of PCs, strengthening the inhibitory output of PC on the next relay neurons including the $\mathrm{VN}$. This increased inhibition to the $\mathrm{VN}$, however, is also possible to enhance the activity of neural circuit via boosting up the rebound activity followed by strong hyperpolarization in the relay neurons (Sekirnjak et al., 2003). Our observations support the former expectation in which the downregulation of intrinsic excitability occurred with the synaptic LTD in PCs. These results are parallel with the prediction of which cerebellum-dependent motor learning is associated with the reduced cortical activity (Lev-Ram et al., 2003). Recently, Yang and Santamaria (2016) reported that synaptic LTD induction potentiates PC excitability showing the opposite to our observation. The investigators paired PF stimulation and the somatic depolarization instead of CF stimulation with $1 \mathrm{~Hz}$ to induce the LTD. This protocol may trigger synaptic and intrinsic plasticity via activation of the different intracellular signaling from our experimental condition. In contrast, we delivered PF and CF stimulation with two types of protocol to reflect more physiological circumstance and applied the LTD-Pseudo induction protocol to exclude bias of long-term recording. Conjunctive activation of PF and CF successfully induces not only the synaptic LTD, but also the LTD-IE. Considering that the LTP-IE was accompanied by LTP induction in PCs (Belmeguenai et al., 2010), our results provide insight into the bidirectional modulation of intrinsic excitability in a stimuli-pattern-specific manner. The same polarity of plasticity between synaptic strength and intrinsic excitability may emphasize the decreased or potentiated synaptic current by newly tuned membrane excitability, by which total net output of the neurons are generated through multiplication of the synaptic current and intrinsic excitability. Surprisingly, temporal EPSP summation and SFA are not altered by the induction of plasticity, indicating that PCs consistently maintain the strategy to process information and filter the output signal after LTD-IE occurs. Together, our observations suggest functional roles of the PC intrinsic plasticity as the amplifier within a motor learning circuitry.

\section{Underlying mechanism of LTD-IE}

Intrinsic plasticity, including LTP- and LTD-IE, can be triggered by synaptic stimulation inducing LTP and LTD, respectively (Belmeguenai et al., 2010), indicating that synaptic plasticity and intrinsic plasticity share molecular cascades for plasticity induction. Coupled activation by PF and CF is the prerequisite for cerebellar LTD induction, which is established through the positive feedback loop of PKC-MAPK signaling. By using an intracellular $\mathrm{Ca}^{2+}$ chelator and an inhibitor peptide of $\mathrm{PKC}$, we confirmed that intracellular $\mathrm{Ca}^{2+}$ signaling plays a central role in 
Table 2. Active properties of action potential before and after LTD induction in floccular PCs

\begin{tabular}{|c|c|c|c|c|c|c|}
\hline & $V_{\text {threshold }}(\mathrm{mV})$ & AP amplitude (mV) & fAHP (mV) & $\mathrm{mAHP}(\mathrm{mV})$ & FWHM (ms) & $R_{\text {in }}(\mathrm{M} \Omega)$ \\
\hline \multicolumn{7}{|l|}{ FL-LTD } \\
\hline Baseline & $-47.6 \pm 1.2$ & $80.1 \pm 3.3$ & $10.5 \pm 0.6$ & $8.7 \pm 0.9$ & $0.29 \pm 0.01$ & $74.8 \pm 3.2$ \\
\hline$t=20$ & $-48.6 \pm 1.3$ & $76.9 \pm 2.7$ & $10.7 \pm 1.0$ & $5.1 \pm 1.0^{* * *}$ & $0.31 \pm 0.02$ & $61.3 \pm 4.1^{*}$ \\
\hline$t=40$ & $-49.5 \pm 1.2^{*}$ & $78.5 \pm 3.2$ & $11.3 \pm 0.8$ & $4.4 \pm 1.1^{* * *}$ & $0.31 \pm 0.02$ & $58.2 \pm 3.9 * * *$ \\
\hline
\end{tabular}

Asterisks in here marked by post hoc Tukey's test after one-way RM ANOVA. ${ }^{*} p<0.05,{ }^{* * *} p<0.001$

LTD-IE induction and synaptic and intrinsic plasticity has a common pathway. In addition, LTD is mediated by the more complex interaction between kinases and phosphatases. These interactions have also implicated in the intrinsic plasticity through ion channel regulation (Cudmore and Turrigiano, 2004; Brager and Johnston, 2007; Belmeguenai et al., 2010; Steinert et al., 2011; Shim et al., 2016). We observed the alteration of AP firing properties, such as reduction of $R_{\text {in }}$ and mAHP, reflecting the changes in the ion channel conductance and/or expression level. Because it has been shown that the mAHP correlates to the SK channels, we tested whether SK channels are required for LTD-IE though the bath application of apamin (Pedarzani et al., 2005; Kato et al., 2006). The involvement of SK channels in LTD-IE because attenuation of $\mathrm{mAHP}$ is also shown without changes in excitability when unpaired synaptic stimuli are delivered (LTDPseudo) and application of apamin, an SK channel inhibitor, is ineffective for LTD-IE induction. LTD-IE induction also results in the reduction of $R_{\mathrm{in}}$. This change is derived from the activitydependent modulation of ion channels such as voltage-gated $\mathrm{K}^{+}$ channels and hyperpolarization-activated cyclic nucleotide gated channels (Hyun et al., 2013; Shim et al., 2016), rather than SK channels. Given the downregulation of the intrinsic excitability, even though the voltage threshold is shifted to hyperpolarization after LTD induction, changes of $R_{\text {in }}$ may have a larger impact on the intrinsic excitability via reducing voltage deflection in response to the given current. In this study, we observed intrinsic plasticity accompanied by synaptic LTD and concluded that synaptic and intrinsic plasticity are mediated by the similar signaling cascades. It remains to be elucidated what precise mechanism underlies intrinsic plasticity.

\section{Intrinsic plasticity in motor learning circuitry}

What are the physiological consequences of the intrinsic plasticity of cerebellar PCs? The neural plasticity occurs in the cerebellar cortex and the $\mathrm{VN}$ are important components for VOR learning (Boyden et al., 2004; Ito, 2013). Given that cerebellar PCs are the only neuron connecting to the $\mathrm{VN}$ from the cerebellar cortex, the activity-dependent modulation of the PC excitability would have physiological significance for motor learning. A mathematical model demonstrated the serial process for long-term memory consolidation of eye movement learning (Yamazaki et al., 2015). According to the study, depression of synaptic weight between $\mathrm{PF}-\mathrm{PC}$ precedes the changes in synaptic strength between MF and $\mathrm{VN}$ in the learning phase. Interestingly, the synaptic weight of MF-VN synapses continuously strengthens in the posttraining phase for several hours even though PF-PC LTD is recovered after training. This theoretical expectation is consistent with previous studies reporting that the posttraining phase is important for consolidation (Kassardjian et al., 2005; Okamoto et al., 2011). Intrinsic plasticity, therefore, may precisely instruct the neural plasticity in the VN neurons to consolidate the memory. In addition, our observation suggests the bidirectional modulation of intrinsic excitability in response to synaptic plasticity. This bidirectionality of the intrinsic plasticity of PC may have an essential role in cerebellum-dependent motor learning circuitry because the polarity of synaptic plasticity are selectively engaged with learning paradigms, gain-up and gain-down (Boyden et al., 2006). We hypothesize that $\mathrm{VN}$ neurons receive the learning pattern via the bidirectional modulation of the intrinsic plasticity and synaptic plasticity in the cerebellar cortex. In other words, PCs might collect the information through synaptic plasticity and transfer the information by modifying their excitability.

This engaged behavioral evidence leads to the question whether LTD-IE can be reversed by application of the LTPinducing protocol because neural activity is dynamically regulated in response to the behavioral modification. However, our results show that LTD-IE cannot be reversed into LTP-IE and vice versa. These results might be explained by the following. First, Lev-Ram et al. (2003) described that synaptic plasticity induced by a higher frequency of PF stimulation than $1 \mathrm{~Hz}$ cannot be reversed because the stronger stimuli result in more saturation of plasticity. Second, the total duration of recording and stimulation interval may not be enough to elicit the reversibility. A mathematical model suggested that the change of cerebellar PC output is gradually developed for $2-4 \mathrm{~h}$ after the learning phase (Yamazaki et al., 2015). In addition, the behavioral reversal test described by Boyden and Raymond (2003) indicates that the reversal in excitability might build over a longer time than observed in slice recording. Because intrinsic plasticity may be involved in several behavioral consequences, unrevealed features of the intrinsic plasticity in behavior should be clarified in further studies.

\section{References}

Belmeguenai A, Hosy E, Bengtsson F, Pedroarena C, Piochon C, Teuling E, He Q, Ohtuski G, De Jeu MT, Elgersma Y, De Zeeuw CI, Jorntell H, Hansel C (2010) Intrinsic plasticity complements long-term potentiation in parallel fiber input gain control in cerebellar Purkinje cells. J Neurosci 30:13630-13643. CrossRef Medline

Benda J, Herz AV (2003) A universal model for spike-frequency adaptation. Neural Comput 15:2523-2564. CrossRef Medline

Boyden ES, Raymond JL (2003) Active reversal of motor memories reveals rules governing memory encoding. Neuron 39:1031-1042. CrossRef Medline

Boyden ES, Katoh A, Raymond JL (2004) Cerebellum-dependent learning: the role of multiple plasticity mechanisms. Annu Rev Neurosci 27:581609. CrossRef Medline

Boyden ES, Katoh A, Pyle JL, Chatila TA, Tsien RW, Raymond JL (2006) Selective engagement of plasticity mechanisms for motor memory storage. Neuron 51:823-834. CrossRef Medline

Brager DH, Johnston D (2007) Plasticity of intrinsic excitability during long-term depression is mediated through mGluR-dependent changes in I(h) in hippocampal CAl pyramidal neurons. J Neurosci 27:1392613937. CrossRef Medline

Coesmans M, Weber JT, De Zeeuw CI, Hansel C (2004) Bidirectional parallel fiber plasticity in the cerebellum under climbing fiber control. Neuron 44:691-700. CrossRef Medline

Cudmore RH, Turrigiano GG (2004) Long-term potentiation of intrinsic excitability in LV visual cortical neurons. J Neurophysiol 92:341-348. CrossRef Medline

De Zeeuw CI, Hansel C, Bian F, Koekkoek SK, van Alphen AM, Linden DJ, Oberdick J (1998) Expression of a protein kinase C inhibitor in Purkinje 
cells blocks cerebellar LTD and adaptation of the vestibulo-ocular reflex. Neuron 20:495-508. CrossRef Medline

Galliano E, Gao Z, Schonewille M, Todorov B, Simons E, Pop AS, D’Angelo E, van den Maagdenberg AM, Hoebeek FE, De Zeeuw CI (2013) Silencing the majority of cerebellar granule cells uncovers their essential role in motor learning and consolidation. Cell Rep 3:1239-1251. CrossRef Medline

Gao Z, van Beugen BJ, De Zeeuw CI (2012) Distributed synergistic plasticity and cerebellar learning. Nat Rev Neurosci 13:619-635. CrossRef Medline

Grasselli G, He Q, Wan V, Adelman JP, Ohtsuki G, Hansel C (2016) Activity-dependent plasticity of spike pauses in cerebellar Purkinje cells. Cell Rep 14:2546-2553. CrossRef Medline

Hansel C, de Jeu M, Belmeguenai A, Houtman SH, Buitendijk GH, Andreev D, De Zeeuw CI, Elgersma Y (2006) $\alpha$ CaMKII is essential for cerebellar LTD and motor learning. Neuron 51:835-843. CrossRef Medline

Hyun JH, Eom K, Lee KH, Ho WK, Lee SH (2013) Activity-dependent downregulation of $\mathrm{D}$-type $\mathrm{K}+$ channel subunit $\mathrm{Kv} 1.2$ in rat hippocampal CA3 pyramidal neurons. J Physiol 591:5525-5540. CrossRef Medline

Ito M (1982) Cerebellar control of the vestibulo-ocular reflex around the flocculus hypothesis. Annu Rev Neurosci 5:275-296. CrossRef Medline

Ito M (2013) Error detection and representation in the olivo-cerebellar system. Front Neural Circuits 7:1. CrossRef Medline

Kandel ER, Dudai Y, Mayford MR (2014) The molecular and systems biology of memory. Cell 157:163-186. CrossRef Medline

Kassardjian CD, Tan YF, Chung JY, Heskin R, Peterson MJ, Broussard DM (2005) The site of a motor memory shifts with consolidation. J Neurosci 25:7979-7985. CrossRef Medline

Kato M, Tanaka N, Usui S, Sakuma Y (2006) The SK channel blocker apamin inhibits slow afterhyperpolarization currents in rat gonadotropin-releasing hormone neurones. J Physiol 574:431-442. CrossRef Medline

Konnerth A, Dreessen J, Augustine GJ (1992) Brief dendritic calcium signals initiate long-lasting synaptic depression in cerebellar Purkinje cells. Proc Natl Acad Sci U S A 89:7051-7055. CrossRef Medline

Lev-Ram V, Mehta SB, Kleinfeld D, Tsien RY (2003) Reversing cerebellar long-term depression. Proc Natl Acad Sci U S A 100:15989-15993. CrossRef Medline

Mahon S, Charpier S (2012) Bidirectional plasticity of intrinsic excitability controls sensory inputs efficiency in layer 5 barrel cortex neurons in vivo. J Neurosci 32:11377-11389. CrossRef Medline

Napper RM, Harvey RJ (1988) Quantitative study of the Purkinje cell dendritic spines in the rat cerebellum. J Comp Neurol 274:158-167. CrossRef Medline

Narayanan R, Dougherty KJ, Johnston D (2010) Calcium store depletion induces persistent perisomatic increases in the functional density of $h$ channels in hippocampal pyramidal neurons. Neuron 68:921-935. CrossRef Medline

Nataraj K, Le Roux N, Nahmani M, Lefort S, Turrigiano G (2010) Visual deprivation suppresses L5 pyramidal neuron excitability by preventing the induction of intrinsic plasticity. Neuron 68:750-762. CrossRef Medline

Okamoto T, Shirao T, Shutoh F, Suzuki T, Nagao S (2011) Post-training cerebellar cortical activity plays an important role for consolidation of memory of cerebellum-dependent motor learning. Neurosci Lett 504: 53-56. CrossRef Medline

Pedarzani P, McCutcheon JE, Rogge G, Jensen BS, Christophersen P, Hougaard C, Strøbaek D, Stocker M (2005) Specific enhancement of SK channel activity selectively potentiates the afterhyperpolarizing current IAHP and modulates the firing properties of hippocampal pyramidal neurons. J Biol Chem 280:41404-41411. CrossRef Medline

Pozzorini C, Naud R, Mensi S, Gerstner W (2013) Temporal whitening by power-law adaptation in neocortical neurons. Nat Neurosci 16:942-948. CrossRef Medline

Safo P, Regehr WG (2008) Timing dependence of the induction of cerebellar LTD. Neuropharmacology 54:213-218. CrossRef Medline

Sekirnjak C, Vissel B, Bollinger J, Faulstich M, du Lac S (2003) Purkinje cell synapses target physiologically unique brainstem neurons. J Neurosci 23 : 6392-6398. Medline

Shim HG, Jang SS, Jang DC, Jin Y, Chang W, Park JM, Kim SJ (2016) mGlu1 receptor mediates homeostatic control of intrinsic excitability through Ih in cerebellar Purkinje cells. J Neurophysiol 115:2446-2455. CrossRef Medline

Steinert JR, Robinson SW, Tong H, Haustein MD, Kopp-Scheinpflug C, Forsythe ID (2011) Nitric oxide is an activity-dependent regulator of target neuron intrinsic excitability. Neuron 71:291-305. CrossRef Medline

Straka H, Vibert N, Vidal PP, Moore LE, Dutia MB (2005) Intrinsic membrane properties of vertebrate vestibular neurons: Function, development and plasticity. Prog Neurobiol 76:349-392. CrossRef Medline

Suvrathan A, Payne HL, Raymond JL (2016) Timing rules for synaptic plasticity matched to behavioral function. Neuron 92:959-967. CrossRef Medline

van Welie I, du Lac S (2011) Bidirectional control of BK channel open probability by CAMKII and PKC in medial vestibular nucleus neurons. J Neurophysiol 105:1651-1659. CrossRef Medline

Wulff P, Schonewille M, Renzi M, Viltono L, Sassoè-Pognetto M, Badura A, Gao Z, Hoebeek FE, van Dorp S, Wisden W, Farrant M, De Zeeuw CI (2009) Synaptic inhibition of Purkinje cells mediates consolidation of vestibulo-cerebellar motor learning. Nat Neurosci 12:1042-1049. CrossRef Medline

Yamazaki T, Nagao S, Lennon W, Tanaka S (2015) Modeling memory consolidation during posttraining periods in cerebellovestibular learning. Proc Natl Acad Sci U S A 112:3541-3546. CrossRef Medline

Yang Z, Santamaria F (2016) Purkinje cell intrinsic excitability increases after synaptic long term depression. J Neurophysiol 116:1208-1217. CrossRef Medline 